\title{
Genital self-mutilation: A challenging pathology (Review)
}

\author{
SORIN LUPU ${ }^{1}$, OVIDIU GABRIEL BRATU ${ }^{2}$, DELIA MIRELA TIT ${ }^{3}$, \\ SIMONA BUNGAU ${ }^{3}$, OCTAVIAN MAGHIAR ${ }^{4 *}$, TEODOR ANDREI MAGHIAR $^{4 *}$, \\ CAMELIA C. SCARNECIU ${ }^{* *}$ and IOAN SCARNECIU ${ }^{6}$ \\ ${ }^{1}$ Clinic of Urology, Brasov Emergency Clinical County Hospital, Brasov 500326; ${ }^{2}$ Clinical Department 3, \\ 'Carol Davila' University of Medicine and Pharmacy, 050474 Bucharest; Departments of ${ }^{3}$ Pharmacy, \\ and ${ }^{4}$ Surgical Disciplines, Faculty of Medicine and Pharmacy, University of Oradea, 3700 Oradea; \\ Departments of ${ }^{5}$ Fundamental, Prophylactic and Clinical Disciplines, and ${ }^{6}$ Medical and Surgical Specialities, \\ Faculty of Medicine, 'Transilvania' University of Brasov, 500019 Brasov, Romania
}

Received May 7, 2021; Accepted June 7, 2021

DOI: $10.3892 / \mathrm{etm} .2021 .10564$

\begin{abstract}
Genital self-mutilation is a pathology that leads to numerous and important discussions, rarely presented in the medical literature. There have been many attempts to explain the reasons behind these medical phenomena, but single cases have been generally reported, making it extremely difficult to draw valid conclusions. It is acknowledged that there are psychotic and non-psychotic causes, from psychiatric problems and sexual identity disorders to cultural or religious reasons, alcohol or recreational drug consumption, unconventional types of sexual satisfaction or self-satisfaction. Recent theories consider self-mutilation as a phenomenon of reducing distress or tension, as an expression of feelings of anger or sorrow. It is believed that $55-85 \%$ of those who have resorted to self-mutilation have at least once in their life tried to commit suicide. There is evidence that early discovery and intervention as well as proper treatment in regards to psychosis can significantly reduce the number of self-mutilation episodes, with a protective role of these individuals. Cases of genital self-mutilation may be considered real medical emergencies, sometimes extremely challenging and accompanied by severe complications. Injury of the genital area is usually accompanied by numerous early or long-term complications due to the marked
\end{abstract}

Correspondence to: Professor Ovidiu Gabriel Bratu, Clinical Department 3, 'Carol Davila' University of Medicine and Pharmacy, 37 Dionisie Lupu, 050474 Bucharest, Romania

E-mail: ovi78doc@yahoo.com

Professor Delia Mirela Tit, Department of Pharmacy, Faculty of Medicine and Pharmacy, University of Oradea, 5 Armatei Romane Street, 3700 Oradea, Romania

E-mail: mirela_tit@yahoo.com

${ }^{*}$ Contributed equally

Key words: genital self-mutilation, rare cases, auto-aggressive behavior, psychotic, non-psychotic, medical emergencies vascular area and to the microbial flora present in this part of the body. The degree of mutilation is an unforeseen aspect that the medical staff may have to encounter during the intervention, sometimes testing their imagination and surgical skills when dealing with such a case. Understanding the causes of these self-aggressive behaviors, which may be life-threatening, is critical and multidisciplinary mobilization is needed after treatment of the acute phases. The outcome of these patients depends on integrated collaborative work. These cases represent a serious reason for frustration for the physicians involved in solving them, and knowledge of these issues is valuable to urologists, psychiatrists and other health professionals.

\section{Contents}

1. Introduction

2. Epidemiology

3. Reasons

4. Discussion

5. Conclusions

\section{Introduction}

Self-mutilation represents voluntary intentional injury focused on the person's own body, without suicidal intent $(1,2)$. Self-mutilation is listed as a symptom of borderline personality disorders $(2,3)$. However, there are experts who consider self-injury as a distinct clinical syndrome (1). Self-mutilation in minor forms is frequently encountered and is dependent on the cultural factors the person experiences and does not cause infirmity. The term of self-mutilation was first used by L.E. Emerson in 1913 (4), and later mentioned by other authors. The topic has become an important one and numerous classifications have been made in an attempt to differentiate between socially accepted and deviant behaviors (1). However, only isolated, single cases have been generally reported, and not multiple cases. Thus, it is extremely difficult to draw valid conclusions (5). 
Genital self-mutilation (GSM) refers to self-aggressive behavior on the genitals, with aggressivity of varying degrees, ranging from the smallest mutilations (blade scratches) to the most serious ones, which constitute urological emergencies (penile or testicular amputation). Once solved, the case requires mandatory psychiatric evaluation and therapeutic approach. However, when these cases occur, they represent a real challenge for urologists and psychiatrists, being isolated cases or repetitive ones $(1,2,5,6)$. The aim of this review emphasizes the main pathological aspects related to genital self-mutilation over the last 120 years.

\section{Epidemiology}

GSM is a rare medical condition generally associated with psychotic phenomena. Several cases associated with various reasons and justifications of religious or sexual nature, many of which are anecdotal in character, have been reported in non-psychotic patients. Approximately 110 cases in men have been described in the literature (7-9). This pathology has been described since ancient Rome, Roman priests considering it as the supreme sacrifice of sexual life. The Greeks described the so-called Eshmun complex that comes from the Phoenician god (with the same name) of healing and the tutelary god of Sidon who castrated himself to avoid the advances of the goddess Astronae (10). Numerous cases of self-mutilation for religious reasons have been described in other cultures as well (11).

It is clear that the number of cases of genital self-mutilation is under-reported. Genital self-mutilation has been reported in both sexes, regardless of culture, religion and/or ethnic group (10). The first case of genital self-mutilation in the modern period was reported in 1846 , but the first scientific description belonged to Stroch in $1901(12,13)$.

In recent years, the number of reported cases has increased. Until 1977 only 51 cases were reported in the English medical literature (14). In 1996, Nakaya reviewed 110 cases published between 1979 and 1993 (8). Nevertheless, the number of cases published in the literature concerning this issue is extremely low (15). Thus, the actual prevalence is difficult to establish. The severity of self-mutilation in the reported cases is also different as indicated in a study published in 2009 which showed a prevalence of 1 case in 4 million individuals, but referring only to cases of severe self-mutilation (5).

According to recent studies, there is no clear differentiation in the prevalence of self-mutilation with regard to sex of the patients $(5,10,15)$. Some evidence has shown that this behavior is more common among men (10). By contrast, other studies have shown that this behavior is more common among women $(1,7,16)$. There are many controversies with regard to the age of those who resort to self-mutilation. Certain studies have shown a prevalence of self-mutilating behavior in 10-15\% of healthy children aged 9-18 months (considered pathological after the age of 3) (1), and a higher prevalence among young individuals (17). Other authors have reported cases in patients aged 6-66 years $(10,18)$. It is generally believed that those who resort to self-mutilation are individuals of varying degrees of intellectual development, of any sexual orientation, in marital relationships or not (10).

\section{Reasons}

The reasons behind self-mutilation may vary greatly: From personal crises related to mental problems (such as sexual identity, mental illness, body dysmorphia) to non-psychotic problems (social reasons, the desire to change one's sex, sexual arousal and religious beliefs) (19). Most GSM cases are categorized as being determined by psychiatric reasons as this behavior is considered unconventional (20). A study revealed that in a group of 53 cases of GSM, 87\% of the patients were psychotic and $13 \%$ were non-psychotic (9). In addition, it was shown that in a group of 14 self-mutilated patients $65 \%$ of the patients were psychotic and $35 \%$ were non-psychotic (19). Certain authors have reported fewer psychosis-related cases $(15,21)$, the more common reasons being related to the disturbance of sexual identity. Cases involving psychoses may be due to functional psychoses or due to brain injuries. Individuals with an increased risk of self-mutilation are those with borderline personality disorder, bipolar persons (22), persons suffering from depression (23), or schizophrenia (24). Interestingly, GSM may be the first and only manifestation of a psychiatric condition $(10,25)$, infantile condition, autism or mental retardation (26).

Previous findings have shown that alcohol or various recreational drug consumption represent major risk factors for self-mutilation $(1,27)$. As far as the consumption of recreational drugs is concerned, the main substances involved in these cases are cocaine, cannabis and amphetamine (27). In a study published in 1993, Aboseif et al showed that, 55\% of those who repeatedly resorted to self-mutilation had a history of drug addiction and $31 \%$ had a history of alcohol consumption (19).

Non-psychotic cases typically include personality disorders, sexual conflicts or feelings of guilt, transvestism, unconventional forms of sexual arousal, various complex types of cultural or religious beliefs $(10,19)$. Cases related to different states of sexual arousal and trans-sexualism are considered to be frequent while those related to certain religious beliefs are mainly generated by the assignment of negative connotations to sexuality (19). Subjects who associate sexual conflicts with feelings of guilt in this regard seem to resort to self-mutilation much more frequently than other subjects (8).

Previous findings have shown that individuals who resort to self-mutilation are generally in a psychotic or intoxicated state (with alcohol or recreational drugs) when they self-mutilate $(7,9,21)$. Subjects with a major risk of self-mutilation (even severe) are those in a first episode of schizophrenia (characterized in particular by disillusionment associated with religious or body-related disappointments) (5). For this purpose, the term of Klingsor Syndrome is also used to describe genital self-mutilation due to religious delusions (28). There are authors who recommend the use of this term in all cases of genital self-mutilation caused by psychotic disorders (28) (the name comes from a character in Richard Wagner's Parsifal, namely Klingsor, a magician who resorts to self-castration in order to keep away from his carnal lust). Certain authors divide the reasons for self-mutilation in three categories: Psychotic (especially schizophrenia), tranvestism, and complex reasons of religious or cultural nature (7). However, other theories consider self-mutilation as 
a phenomenon of reducing distress or tension, as a form of expressing feelings of anger or sorrow (29).

Cases of self-mutilation are frequently encountered in prisoners and present multiple causes (30). Self-mutilation can also be associated with suicide attempts. It is believed that $55-85 \%$ of those who have resorted to self-mutilation have at least once in their life tried to commit suicide (2). Although numerous explanations or theories have been offered to explain self-mutilation, a clear conclusion remains to be drawn owing to this pathology being extremely serious and complex. Several predisposing factors for self-mutilation have been suggested including the absence of a male dominant figure during early childhood; effeminate behaviors during childhood; a possessive, dominant, highly protective mother causing the son's masochistic behaviors or unresolved sexual conflicts; rejection, denial of one's body or certain parts of the body (especially the genital organs); feelings of guilt; marked anxiety (18).

Several genetic disorders that obviously predispose subjects to self-mutilation should also be mentioned. The Lesch-Nyhan syndrome is a rare genetic disorder caused by an anomaly on the X-chromosome that occurs almost exclusively in males (31). The Cornelia de Lange syndrome is a rare genetic disorder caused by the mutations of the NIPBL gene on the short arm of chromosome 5 and of SMC1L1 on $\mathrm{X}$-chromosome $(32,33)$. These rare syndromes are associated with severe mental retardation and pose a major risk of self-mutilation (34).

\section{Discussion}

Very few cases of GSM have been published by the specialized literature, being particularly associated with psychotic disorders, ranging from patients with functional psychoses to patients with brain injury (35). Psychotic patients at risk for genital self-mutilation are patients with personality disorders, delusions (especially religious ones), depression, childhood trauma or severe deprivation, or severe feelings of guilt of a sexual nature $(1,10,11,15,20)$. Non-psychotic cases are generally associated with behavioral disorders, with certain sexual behaviors (such as transvestism during the 'difficult' waiting period for sex reassignment surgery from male to female or exaggerated autoerotism) (36), or reasons of a religious nature (including sacrifices) $(11,15,20)$.

Self-mutilation is also encountered in certain situations that present hallucinations, especially caused by the consumption of psychogenic substances. Excessive alcohol intake and/or narcotics were also identified in $54 \%$ of the studied cases $(19,35)$. Another study showed that self-mutilation is much more severe in patients with feelings of sexual guilt (8). The literature presents the case of a boy who began to self-mutilate himself at the age of 6 . By the age of 35 he had already amputated his penis, testicles and scrotum (18). This is considered the most severe sexual self-mutilation $(10,18)$. The psychopathological explanation of these cases includes psychoanalytic, cognitive and neurochemical models that need to be further investigated. Frustration, impulsiveness, aggressiveness that occur at some point in these patients may be determinant factors in triggering such behaviors. The sexual or aggressive needs cannot be solved otherwise. For a better understanding of patient motive, special attention must be paid to the part of the body patients focused on since it may have a symbolic significance. Self-mutilation cases may be considered real medical emergencies, sometimes extremely challenging and with severe complications. There are cases that are discovered accidentally and pose no difficulty or cases that are extremely serious that put the patient's life in danger (bleeding, abscess, or septic states). Injury of the genital area is usually accompanied by numerous early or long-term complications due to the marked vascular area and to the microbial flora present in this part of the body. Proper diagnosis requires complete clinical examination and a proper anamnesis. Paraclinical and imaging investigations are often imposed and provide additional information.

The main purpose of the treatment is to solve the complications caused by self-mutilation, to perform reconstructions (re-implantations or skin grafts), to remove the foreign body/bodies, to treat all the complications caused by these behaviors and to prevent the onset of other complications (especially infectious ones). Managing such cases requires much tact, skill and professionalism on the part of all those involved, without judging patients or showing inappropriate feelings or behaviors towards these persons who already feel guilty and ashamed concerning the situation. The optimal conditions for examination, diagnosis and treatment must be provided, implying the necessary intimacy. Not only are emergency measures required, but long-term measures are imperative. After treating the acute phase, psychiatric evaluation is mandatory in order to reveal the reasons that have led to such behaviors and to prevent relapses (37).

Of note is that such cases are judged and reported in different cultures, with different beliefs and behaviors, raising important issues of interpretation. Besides the current situation caused by the emergency itself, by the early complications and by the long-term complications of self-mutilation, the case requires much tact in handling, empathic management, no sarcastic reactions or behaviors, disgust or hostility from the medical staff. Besides the multidisciplinary approach of the case, psychiatric counselling and evaluation are mandatory as they are the only ones that can provide explanation and hope in these often desperate situations. After treating such cases and going through such experiences with the patient, the physician becomes aware of the fact that correct and sustained psychiatric counselling can lead to a more effective approach with regard to the patient, of such situations, to less self-aggressive and 'wiser' behaviors since the complete renunciation of these behaviors is almost illusory.

Evidence suggest that early discovery and intervention as well as proper treatment in psychosis can significantly reduce the number of self-mutilation episodes, with protective role (5). Besides medical challenges, possible forensic implications of these cases should be considered since there may be situations when the doctor is confronted with issues related to sexual assault, confidentiality, reporting or not to other persons or authorities, or even obtaining patient informed consent. Certain authors recommend obtaining approval for setting up an electronic medical database on rare psychiatric cases in order to draw some pertinent conclusions after analyzing all aspects, to identify certain factors, and to reduce the 
number of self-mutilation episodes (5). All this is crucial due to the fact that GSMs are generally reported as isolated cases or limited series of cases $(38,39)$. However, understanding the causes of these self-aggressive behaviors, which may be life-threatening, is critical and multidisciplinary mobilization is needed after the treatment of the acute phases (40). The outcome of these patients depends on integrated collaborative work. Prevention of such behaviors can also occur especially in patients with pre-existing psychiatric problems who are at risk for self-mutilation mainly. These cases represent a serious reason for frustration for the physicians involved in solving them because they are often refractory to treatment and repetitive and involve high costs (40). In major depression, GSM may be considered a suicide attempt and the act may be attributed to a coping mechanism associated with the symbolic expression of emotions, especially feelings of guilt. Other authors attribute GSM as playing an anti-suicidal role by regulating the dysphoric element (41).

Dysregulation of serotonin transmission in the CNS (central nervous system) is reported to be related to different psychiatric disorders in humans, including depression, impulsive aggression and anxiety disorders (42-44). In depression, depletion of serotonin is linked to aggression, facilitating suicide or self-mutilation.

\section{Conclusions}

Patients who come to the hospital for self-mutilation, especially at the genital level, are rare in current practice. Although motives and reasons vary from psychotic and non-psychotic reasons, psychiatric problems or sexual identity disorders to cultural or religious reasons, alcohol or recreational drug consumption, unconventional forms of sexual satisfaction or self-satisfaction, the management of those patients is a challenging problem and requires interdisciplinary collaboration between urologists, surgeons, psychiatrists, psychologists and other health staff. The staff need to be prepared to manage such cases with tact and professionalism while taking care in view of all the possible legal consequences. After resolving emergency measures, long-term therapy is mandatory for all the patients in order to resolve the problems that led the patient to respond or react with self-harm.

\section{Acknowledgements}

Not applicable.

\section{Funding}

No funding was received.

\section{Availability of data and materials}

All information provided in this review is documented by relevant references.

\section{Authors' contributions}

SL, OGB, DMT, SB and IS collected, analyzed and interpreted the literature data. SL, OGB, DMT, SB, OM, TAM, and CCS made substantial contributions to the conception of the work and interpretation of data; also, they drafted the manuscript and were major contributors in writing the manuscript. All authors read and approved the final manuscript to be published. All the authors agreed to be accountable for all aspects of the work in ensuring that questions related to the accuracy or integrity of any part of the work are appropriately investigated and resolved.

\section{Ethics approval and consent to participate}

Not applicable.

\section{Patient consent for publication}

Not applicable.

\section{Competing interests}

The authors declare that they have no competing interests.

\section{References}

1. Baguelin-Pinaud A, Seguy C and Thibaut F: Self-mutilating behaviour: A study on 30 inpatients. Encephale 35: 538-543, 2009 (In French).

2. Shoib S, Mushtaq R, Arif T and Hijaz SW: Self-mutilation of genitalia masquerading as suicide: A rare case report. J Psychiatry 17: 115, 2014.

3. Gilman SL: From psychiatric symptom to diagnostic category: Self-harm from the Victorians to DSM-5. Hist Psychiatry 24: 148-165, 2013.

4. Emerson LE: The case of miss A: A preliminary report of a psychoanalytic study and treatment of a case of self-mutilation. Psychoanal Rev 1: 41-54, 1913.

5. Large M, Babidge N, Andrews D, Storey P and Nielssen O: Major self-mutilation in the first episode of psychosis. Schizophr Bull 35: 1012-1021, 2009.

6. Feldman MD: The challenge of self-mutilation: A review. Compr Psychiatry 29: 252-269, 1988.

7. Yadukul S, Udaya Shankar BS and Shivakumar BC: A case of genital self-mutilation committed before suicide. Egypt J Forensic Sci 5: 70-72, 2015.

8. Nakaya M: On background factors of male genital self-mutilation. Psychopathology 29: 242-248, 1996.

9. Greilsheimer $\mathrm{H}$ and Groves JE: Male genital self-mutilation. Arch Gen Psychiatry 36: 441-446, 1979.

10. Eke N: Genital self-mutilation: There is no method in this madness. BJU Int 85: 295-298, 2000.

11. Rao KN, Sudarshan CY and Begum S: Self-injurious behavior: A clinical appraisal. Indian J Psychiatry 50: 288-297, 2008.

12. Kharbach Y, Amiroune D, Ahsaini M, Bout A, Riyach O, Stuurman-Wieringa RE, Mellas S, Tazi MF, Khallouk A, El Fassi MJ, et al: Penile self-mutilation preceded by bizarre delusions: Two case reports. J Med Case Rep 8: 246, 2014.

13. Stroch D: Self-castration. JAMA 36: 270, 1901.

14. Evins SC, Whittle T and Rous SN: Self-emasculation: Review of the literature, report of a case and outline of the objectives of management. J Urol 118: 775-776, 1977.

15. Mago V: Male genital self-mutilation. Indian J Psychiatry 53: 168-169, 2011.

16. Fox C and Hawton K: Deliberate Self-harm in Adolescence. London, Jessica Kingsley Publishers, 2004.

17. Blacker KH and Wong N: Four cases of autocastration. Arch Gen Psychiatry 8: 169-176, 1963.

18. Mahadevappa N, Kochhar G, Vilvapathy KS, Dharwadkar S and Kumar S: Self-inflicted foreign bodies in lower genitourinary tract in males: Our experience and review of literature. Urol Ann 8: 338-342, 2016.

19. Aboseif S, Gomez R and McAninch JW: Genital self-mutilation. J Urol 150: 1143-1146, 1993. 
20. Becker $H$ and Hartmann $U$ : Genital self-injury behavior-phenomenologic and differential diagnosis considerations from the psychiatric viewpoint. Fortschr Neurol Psychiatr 65: 71-78, 1997 (In German).

21. Joyce PR, Light KJ, Rowe SL, Cloninger CR and Kennedy MA: Self-mutilation and suicide attempts: Relationships to bipolar disorder, borderline personality disorder, temperament and character. Aust N Z J Psychiatry 44: 250-257, 2014.

22. Gardner DL and Cowdry RW: Suicidal and parasuicidal behavior in borderline personality disorder. Psychiatr Clin North Am 8: 389-403, 1985.

23. Rao KN, Bharathi $\mathrm{G}$ and Chate S: Genital self-mutilation in depression: A case report. Indian J Psychiatry 44: 297-300, 2002.

24. Large M, Andrews D, Babidge N, Hume F and Nielssen O: Self-inflicted eye injuries in first-episode and previously treated psychosis. Aust N Z J Psychiatry 42: 183-191, 2008.

25. Simpson MA: Self-mutilation. Self-injurious behavior: A clinical appraisal. Br J Hosp Med 16: 430-438, 1976.

26. Greydanus DE and Shek D: Deliberate self-harm and suicide in adolescents. Keio J Med 58: 144-151, 2009.

27. Ahsaini M, Tazi F, Khalouk A, Lahlaidi K, Bouazzaoui A Stuurman-Wieringa RE, Elfassi MJ and Farih MH: Bilateral testicular self-castration due to cannabis abuse: A case report. J Med Case Rep 5: 404, 2011.

28. Schweitzer I: Genital self-amputation and the Klingsor syndrome. Aust N Z J Psychiatry 24: 566-569, 1990.

29. Israel JA and Lee K: Amphetamine usage and genital self-mutilation. Addiction 97: 1215-1218, 2002.

30. Kaba F, Lewis A, Glowa-Kollisch S, Hadler J, Lee D, Alper H, Selling D, MacDonald R, Solimo A, Parsons A and Venters H: Solitary confinement and risk of self-harm among jail inmates. Am J Public Health 104: 442-447, 2014.

31. Nyhan WL: Lesch-nyhan disease. J Hist Neurosci 14: 1-10, 2005.

32. Boyle MI, Jespersgaard C, Brøndum-Nielsen K, Bisgaard AM and Tümer Z: Cornelia de Lange syndrome. Clin Genet 88: 1-12, 2015.
33. Pié J, Gil-Rodríguez MC, Ciero M, López-Viñas E, Ribate MP, Arnedo M, Deardorff MA, Puisac B, Legarreta J, de Karam JC, et al: Mutations and variants in the cohesion factor genes NIPBL, SMC1A, and SMC3 in a cohort of 30 unrelated patients with Cornelia de Lange syndrome. Am J Med Genet A 152A: 924-929, 2010.

34. Wraight WM, Belcher HJ and Critchley HD: Deliberate self-harm by insertion of foreign bodies into the forearm. J Plast Reconstr Aesthet Surg 61: 700-703, 2008.

35. Nerli RB, Ravish IR, Amarkhed SS, Manoranjan UD, Prabha V and Koura A: Genital self-mutilation in nonpsychotic heterosexual males: Case report of two cases. Indian J Psychiatry 50: 285-287, 2008

36. Money J and De Priest M: Three cases of genital self-surgery and their relationship to transexualism. J Sex Res 12: 283-294, 1976.

37. Lucerna A: Foreign body insertions: A review. Treating patients who present with foreign body insertions requires a nonjudgmental and open-minded approach. Emerg Med 49: 315-319, 2017.

38. Khan MK, Usmani MA and Hanif SA: A case of self amputation of penis by cannabis induced psychosis. J Forensic Leg Med 19: 355-357, 2012.

39. Park SC, Park YC and Choi J: A case of Klingsor syndrome in Korea. Psychiatry Clin Neurosci 65: 680-681, 2011.

40. Poynter BA, Hunter JJ, Coverdale JH and Kempinsky CA: Hard to swallow: A systematic review of deliberate foreign body ingestion. Gen Hosp Psychiatry 33: 518-524, 2011.

41. Simopoulos EF and Trinidad AC: Two cases of male genital self-mutilation: An examination of liaison dynamics. Psychosomatics 53: 178-180, 2012.

42. Coccaro EF, Kavoussi RJ and Hauger RL: Serotonin function and antiaggressive response to fluoxetine: A pilot study. Biol Psychiatry 42: 546-552, 1997.

43. Maria Alexandra Stanescu A, Totan A, Mircescu D Diaconescu S, Gabriel Bratu O, Fekete L, László Fekete G, Boda D and Cristina Diaconu C: Assessment of suicidal behavior in dermatology (Review). Exp Ther Med 20: 73-77, 2020.

44. Farcas C, Botea V, Popescu R, Dinu M, Bratu O, Spînu D and Mischianu D: Penile fracture-clinical case presentation. Romanian J Military Med 118: 45-48, 2015. 